\title{
Immune responses link parasite genetic diversity, prevalence and plumage morphs in common buzzards
}

\author{
Nayden Chakarov ${ }^{1,2}$ (D) Martina Pauli $^{1} \cdot$ Oliver Krüger $^{1}$
}

Received: 22 February 2016/Accepted: 31 October 2016/Published online: 4 November 2016

(C) The Author(s) 2016. This article is published with open access at Springerlink.com

\begin{abstract}
The evolution and maintenance of conspicuous phenotypic polymorphisms has challenged evolutionary ecologists for centuries. Polymorphisms in pleiotropic cascades or genetic linkage may lead to correlations of life history traits such as immunity, parasite infection levels, pigmentation and lifetime reproductive success. The common buzzard Buteo buteo is a bird of prey occurring in several plumage morphs, which differ in pigmentation and in the prevalence, infection intensity and clone composition of their most common blood parasite, as well as in ectoparasite infestation levels. Buzzard morphs are heritable and exhibit a heterozygote advantage where intermediates have higher lifetime reproductive success (LRS). We explored the hypothesis that the differences in pigmentation also correspond to differences in immunity. We hence compared an inducible adaptive and an innate constitutive immune response between the buzzard plumage morphs. The increase of specific anti-tetanus antibodies after vaccination was explained by the morph of the nestling's mother and was highest in offspring of intermediate mothers. Additionally, nestlings with higher humoral response were less infected with blood parasites and, if infected, harboured a lower genetic diversity of these parasites. The phytohaemagglutinin-induced skin swelling, a complex of cellular inflammatory responses, was lowest in intermediate nestlings. The higher LRS of intermediate buzzards suggests that the cellular immunity is an inferior fitness determinant compared to humoral immunity. The strength of immune responses was not linear along the melanisation gradient, indicating that there is most likely no simple genetic correlation between immune responses and plumage morphs.
\end{abstract}

Nayden Chakarov

az.nayden@gmail.com

1 Department of Animal Behaviour, Bielefeld University, PO Box 1001 31, 33501 Bielefeld, Germany

2 Department of Biology, Molecular Ecology and Evolution Lab, Ecology Building, Lund University, 22362 Lund, Sweden 
Keywords POMC $\cdot$ Maternal effects $\cdot$ Natural antibodies $\cdot$ Carnus $\cdot$ Delayed-type hypersensitivity

\section{Introduction}

Understanding the mechanisms of polymorphism maintenance under selection continues to be a significant challenge for evolutionary biologists. Often, the most conspicuous polymorphic traits are assumed to be the most important, while pleiotropic effects and correlated phenotypes can conceal concomitant traits which are under stronger selection. For example, melanin polymorphisms are widely distributed in many clades, but their adaptive significance still remains unclear (Fowlie and Krüger 2003; Roulin 2004). The pro-opiomelanocortin (POMC) pleiotropy has been suggested to cause positive correlations between pigmentation, humoral immunity and anti-inflammatory responses, since the involved hormones can regulate both the melanisation and systemic inflammation in mice (Ducrest et al. 2008). Several studies have indeed found correlations between melanin pigmentation and immune responses in wild vertebrates as a possible consequence of the POMC pleiotropy. However, the precise molecular link to the humoral response is so far unknown and the documented patterns are, at least partially, contradictory (Gangoso et al. 2011, 2015; Jacquin et al. 2011, 2013; Lei et al. 2013; Roulin et al. 2000).

The immune response is the final and most sophisticated layer of defence which hosts have against parasites and pathogens (Janeway et al. 2005). Immune defence components can be separated into cellular and humoral or innate and adaptive (Schmid-Hempel and Ebert 2003). Different parts of the immune system have evolved to deal with different types of parasites, but immune trade-offs, as well as trade-offs with other life-history components can arise. Additionally, discrete morphs within a species can be differently constrained by such trade-offs (Gasparini et al. 2009a, c; Krasnov et al. 2005; Lochmiller and Deerenberg 2000; McDade et al. 2016; Moret 2003; Norris and Evans 2000). Therefore, different individuals may differ in susceptibility and tolerance to different types of parasites (Svensson and Råberg 2010).

One example of a pigmentation polymorphism with potential links to immunity is the common buzzard Buteo buteo. This bird of prey is found throughout Eurasia and has a variable expression of melanins with three coexisting, but easily distinguishable plumage morphs: dark, intermediate and light (Glutz von Blotzheim et al. 1971). The polymorphism behaves as if encoded by a single autosomal locus, possibly involved in the POMC pleiotropic cascade. Plumage morph seems to be inherited in a Mendelian fashion with both parents contributing "half" of each offspring's morph phenotype (Krüger et al. 2001). The buzzard polymorphism could be sustained through a heterozygote advantage, since for both sexes in a population in Germany, intermediate buzzards showed up to twice higher lifetime reproductive success than dark and light individuals (Chakarov et al. 2008; Krüger et al. 2001). Buzzard morphs differ in multiple other traits, such as breeding phenology, behaviour and habitat use (Boerner and Krüger 2009; Chakarov et al. 2013; Krüger 2002). Yet, among the best candidates to affect their fitness in the long-term are the differences between morphs in parasite infections. Previously, we have shown that dark nestlings are most heavily infested by the common ectoparasite Carnus haemapterus, a blood-sucking fly. In contrast, the most common blood parasite Leucocytozoon has lowest prevalence in intermediate nestlings and highest infection intensities in light nestlings (Chakarov et al. 
2008). Additionally, the genetic similarity of Leucocytozoon infections was best explained by the plumage morph of the hosts' mothers (Chakarov et al. 2015a). Since melanin morph appear to not be spatially segregated (Chakarov et al. unpublished data) these infection patterns suggest that morphs may differ in immunity components responsible for the protection against different parasites and the shaping of parasite communities.

In this study, we test the hypothesis that morphs differ not only in parasite infection patterns, but also in two components of the immune defence. One is the delayed-type hypersensitivity response, caused by an injection of the mitogen phytohaemagglutinin (PHA) into the skin. This response has been shown to correspond to leukocyte profiles and is indicative of the first line of cellular response (Martin et al. 2006). If the POMC pleiotropic cascade influences both melanin production and immune responses to parasites, then we predict (1) that dark nestlings will have the lowest PHA response, while light nestlings should have the highest (Ducrest et al. 2008). Secondly, many infections which have managed to establish themselves in the host are effectively reduced and controlled via specific antibodies (Riley et al. 2006). The POMC-pleiotropy hypothesis predicts a positive correlation between melanisation and proliferation of B-lymphocytes and production of antibodies (Ducrest et al. 2008). Thus we predict that (2) the inducible humoral response, which produces specific antibodies, should be highest in dark nestlings and weakest in light nestlings. The production of maternal antibodies can be influenced by the POMC pleiotropy, similarly to other immunoglobulins (Gasparini et al. 2009b). Since maternal antibodies can affect the immunity of nestlings in their first weeks of life, we consider morph of the mother to be another potential variable explaining the nestling's immune responses (Gasparini et al. 2009b; Jacquin et al. 2013).

The host immune system identifies parasitic antigens which are similar and related more easily than dissimilar ones (Caillaud et al. 2006). Thus, the genetic diversity of parasites may decrease with the strength of the host immune response and host age as a correlate of the former (Ntoumi et al. 1995; Van den Broeck et al. 2014). Since plumage morph is a predictor of prevalence, parasitemia and parasite diversity in buzzards (Chakarov et al. 2008, 2015a), and the humoral response is the most probable intermediate link between morph and infection measures, we predicted that (3) the antibody titres will negatively correlate with infection prevalence, parasitemia and parasite genetic diversity.

\section{Materials and methods}

\section{Study population and field procedures}

The study was carried out between 2010 and 2012 in the same buzzard population where infection differences between buzzard morphs were previously established (Chakarov et al. 2008). It is situated in a $300 \mathrm{~km}^{2}$ study area in Eastern Westphalia, Germany $\left(8^{\circ} 25^{\prime}\right.$ and $52^{\circ} 06^{\prime}$ ). Each year, nests were located between March and May. Nest trees were climbed and nestlings were brought to the ground, tarsus length was measured with a calliper to the nearest $0.1 \mathrm{~mm}$, wing length with a ruler to the nearest $\mathrm{mm}$ and body mass was taken with a Pesola spring balance to the nearest $5 \mathrm{~g}$. The residuals of the body mass-tarsus regression were used as a proxy for current body condition (Roulin 2004; Sternalski et al. 2012). Carnus infestation score was grouped into one of five classes according to the number of underwing and leg pits where infestation signs could be found $(0=$ no infestation to $4=$ all wing and leg pits infested). Morphs of the nestlings and their mother and father 
were recorded. Like many Accipitrid raptors, buzzards are expected to maintain genetic monogamy and have very low rates of extra-pair paternity (Briggs and Collopy 2012; Rudnick et al. 2005; Woolaver et al. 2013), hence the social father is very likely to be the genetic one, too. Blood samples $(50-500 \mu \mathrm{l})$ were taken from the brachial vain of nestlings into a heparinized capillary. A drop of blood was used to prepare blood smears and the rest was centrifuged within $10 \mathrm{~min}$ to separate cells and plasma. The plasma was stored at $-20{ }^{\circ} \mathrm{C}$ until analyses. Blood cells were reconstituted in PBS-EDTA buffer and used for DNA extractions from which nestling sex was determined following a standard protocol (Fridolfsson and Ellegren 1999). After handling, chicks were brought back to the nest. Blood smears were swiftly air dried, fixed in absolute ethanol within $24 \mathrm{~h}$ and stained with Giemsa (Sigma) within 30 days. From these blood smears, 10,000 erythrocytes were scanned under a $500 \times$ magnification to determine Leucocytozoon infection status and infection intensity. Buzzard nests were further observed until feather plumage was complete and the morph of nestling could be confirmed visually. All sampling and experimental treatments were done with permission from the local authority, Kreis Gütersloh, permit nr: 4.5.2-723-Bussard.

\section{Specific antibody response test}

In order to test the strength of the individual humoral response in early nestling age when infection with Leucocytozoon is most probable, we introduced 40 nestlings from 18 broods to a mix of two antigens presumably unknown to them. The mean age of the nestlings was 18 days (10-26). After taking a plasma sample at primary sampling, we injected naïve nestlings with $200 \mu \mathrm{l}$ of tetanus-diphtheria vaccine (Mérieux, $500 \mu \mathrm{l}$ containing $20 \mathrm{IE}$ tetanus and 2 IE diphtheria toxoids) in the pectoral muscle (Råberg et al. 2000; Sandell et al. 2009). Seven days later, the nests were climbed again, and a second blood sample was taken, from which plasma was extracted and stored at $-20{ }^{\circ} \mathrm{C}$. Death of young nestlings due to bad weather, poor food conditions and sibling competition is common in the population (Chakarov et al. 2015b). Six of the vaccine-treated nestlings died between the first and second sampling of plasma, leaving a sample size of 34 chicks. Analyses of the antibody titres were performed with commercially available ELISA plates coated with tetanus or diphtheria toxoids (Demeditec Diagnostics kits DE85101 and DE85111). Handling of pre- and post-treatment sera was performed in tandem. Test sera were diluted $1: 33$ in the kit dilution buffer (PBS/BSA, $0.095 \% \mathrm{NaN}_{3}$ ) in duplicates and $100 \mu \mathrm{l}$ of the solution was pipetted into the wells. The first and last columns of each plate were occupied with four blank and four serial dilutions of a reference plasma sample extracted from a recaptured and previously vaccinated adult buzzard. Plates were incubated for $16 \mathrm{~h}$ at $5{ }^{\circ} \mathrm{C}$ and wells were washed with the kit washing buffer (PBS + Tween 20, all washing steps include three washings with the buffer) and incubated for 90 min with $100 \mu \mathrm{l}$ rabbit antichicken IgG (C2288, Sigma, diluted 1:300 in the kit dilution buffer). Afterwards, wells were washed and incubated 45 min with $100 \mu$ peroxidase-labelled goat anti-rabbit IgG (A6154, Sigma, diluted 1:500). After a further washing, $100 \mu \mathrm{l}$ of substrate (tetramethylbenzidine delivered with the ELISA kits) were added to the wells and incubated for $30 \mathrm{~min}$. Then, $100 \mu \mathrm{l}$ kit stop solution $(1 \mathrm{~N} \mathrm{HCl})$ was added, and the wells and plates were read at $450 \mathrm{~nm}$. Values of individual samples were highly repeatable $(\mathrm{r}>0.99)$. There was a strong correlation between tetanus and diphtheria antibody titres (Pearson: $r=0.623$, $\mathrm{N}=19, p=0.004$ ), similar to previous studies (Svensson et al. 1998). However, values for diphtheria were always very low, potentially because of a lower assay sensitivity or smaller antigen concentration in the vaccine. Because of higher error rate and plasma 
limitations, we did not measure titres for diphtheria antibodies for all birds and only discuss results for tetanus antibodies. The increase in optical density between pre- and post-vaccination sera is further used as a measure of the individual specific anti-tetanus antibody response. The analyses of antibody titres were performed blind to any of the nestling data recorded in the field.

\section{Phytohaemagglutinin challenge}

We used a PHA challenge modified after Smits et al. (1999) as a feasible test of the cellmediated immunity. During primary sampling, a spot on the right wing web of 33 nestlings from 14 broods, aged between 11 and 30 days (mean: 24 days) was marked and its thickness was measured three times. Afterwards, $50 \mu \mathrm{g}$ PHA (L1668, Sigma) dissolved in $100 \mu \mathrm{l}$ PBS were injected into the patagium at the marked location. After $24 \mathrm{~h}( \pm 1 \mathrm{~h})$, nests were climbed again and wing web thickness at the marked location was measured three times. Repeatability of measurement was high $(\mathrm{r}>0.9)$ and the median measure was used in all analyses. The difference in wing web thickness after and before the PHA injection is further used as the response to the stimulus. PHA and vaccination tests were performed on different individuals.

\section{Blood parasite diversity}

An average of $44 \%$ of all buzzard nestlings are infected with Leucocytozoon buteonis (Chakarov et al. 2008). Although mitochondrial lineages are the standard marker of blood parasite diversity (Bensch et al. 2009), the studied buzzard population appears to be infected by one dominant Leucocytozoon lineage. Thus we opted to estimate intraspecific diversity of this parasite species through microsatellites. We estimated the genetic diversity of Leucocytozoon infections by developing 15 microsatellites for Leucocytozoon through high throughput sequencing of Leucocytozoon-enriched buzzard blood (Chakarov et al. 2015a). Microsatellites were PCR-amplified using type-it microsatellite PCR kit (Qiagen) and the resulting fragments were resolved on an ABI 3730 Automated DNA Analyser and analysed using Genemarker 1.95 (SoftGenetics LCC). The maximal number of alleles among any of the microsatellite loci was used as a measure of the number of Leucocytozoon clones, i.e. genetic diversity of the infection within a given host. For complete details see Chakarov et al. (2015a).

\section{Statistical analyses}

The anti-tetanus antibody increase and PHA-induced swelling for individual birds were used as dependent variables in generalized linear mixed effects models, calculated with lmer in the R package lme4. In these models, brood identity and year of sampling were entered as random factors to account for shared genetic background and environmental effects such as food availability, which can influence parts of the immune response (HoiLeitner et al. 2001). Morph of nestlings, maternal and paternal morph were entered in the maximal model as three-level factors. Potential effects of sex and age were accounted for by incorporating in the models sex, and wing length as the best available correlate of age. We present the results from the best models, selected by lowest AICc values (Burnham and Anderson 2002). A separate set of models with similar structure was constructed with Carnus infestation score, Leucocytozoon infection status, infection intensity and number of 
clones as dependent variables, and immunity component measures as explanatory variables. These models had binomial and Poisson error structure and were calculated with glmer in lme4. All means are presented \pm SE.

\section{Results}

\section{Humoral response}

The anti-tetanus antibody response was best explained by the model containing the morph of the nestlings' mother $\left(\chi^{2}=39.032, d f=2, p<0.001\right)$, and the body condition of the nestling during vaccination $(\beta=0.523 \pm 0.078, \mathrm{~F}=29.668, p<0.001)$. As expected from a Mendelian inheritance pattern, the morphs of nestlings and their mothers were correlated $\left(\beta=0.357, \chi^{2}=7.365, p=0.006\right)$. Nevertheless, morph of the nestling did not explain its humoral response $\left(\chi^{2}=0.370, d f=2, p=0.831\right)$. Nestlings of intermediate mothers mounted a stronger anti-tetanus response than offspring of both dark and light mothers (Fig. 1a), and better body condition at the time point of vaccination correlated with a higher antibody titre.

\section{PHA-response}

The PHA-induced swelling was best explained by the nestling morph $\left(\chi^{2}=10.881\right.$, $d f=2, p=0.004$, Fig. 1b). Intermediate nestlings showed a significantly lower cellular response than both dark and light nestlings. Neither body condition before PHA injection or one day after, nor the difference in body mass between both measurements correlated with the PHA-induced swelling.

\section{Parasite prevalence and diversity}

Nestlings infected with Leucocytozoon had a significantly lower humoral response than nestlings which were not infected $\left(\chi^{2}=4.428, d f=1, p=0.035\right)$. The number of Leucocytozoon clones in infected nestlings was negatively correlated with the response against tetanus antigens $\left(\chi^{2}=7.256, d f=1, p=0.007, \mathrm{n}=16\right.$, Fig. 2$)$ and positively correlated with age $\left(\beta=0.096+0.040, \chi^{2}=6.367, d f=1, p=0.012\right)$. These results remained qualitatively unchanged when nestling morph was included in the explanatory models and this variable did not significantly explain neither Leucocytozoon infection status nor Leucocytozoon clone number. Neither the induced antibody titre, nor the PHA-response explained the Carnus haemapterus infestation score.

\section{Discussion}

We showed that two components of the immune response are associated with plumage morphs of the common buzzard. The strength of the humoral response also explained the infection status with blood parasites and their genetic diversity.

The differences between morphs did not correspond to our expectations based on a possible POMC pleiotropic cascade, linearly correlating melanisation and the measured immune responses (Ducrest et al. 2008). Surprisingly, and in contrast to our prediction (1), 

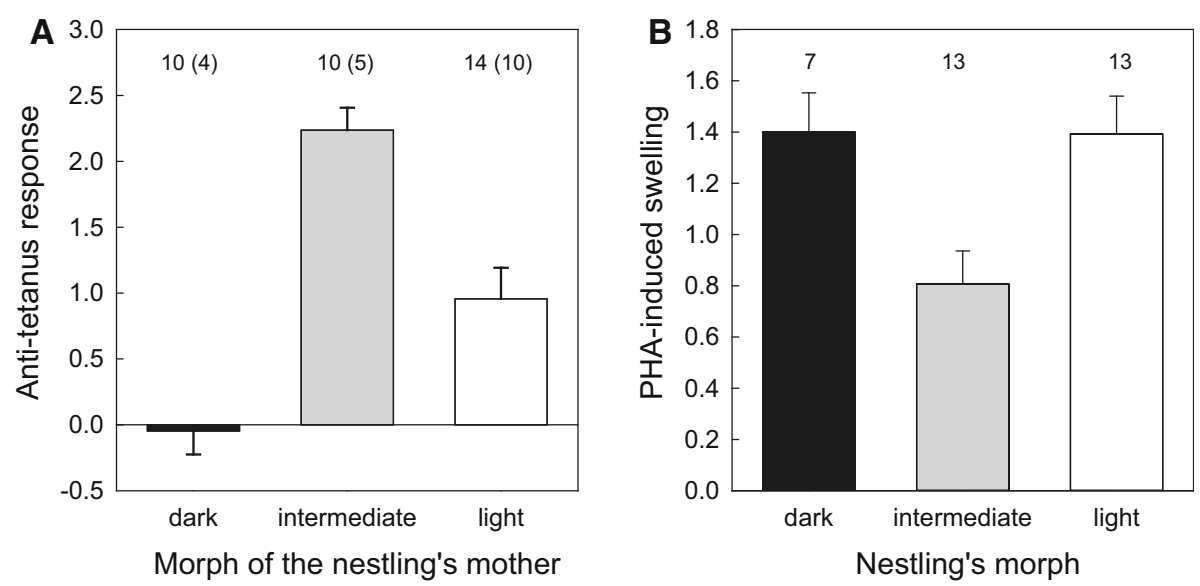

Fig. 1 a Vaccine-induced increase of tetanus antigen binding ( \pm SE) of offspring with mothers of different morphs. Offspring sample sizes with corresponding number of mothers in parentheses are given above bars. b Differences between plumage morphs of nestlings in swelling $( \pm \mathrm{SE})$ induced $24 \mathrm{~h}$ after injection of phytohaemagglutinin (PHA). Sample sizes are given above bars

Fig. 2 Number of Leucocytozoon clones in common buzzard nestlings against antibody response to an experimental challenge with tetanus vaccine

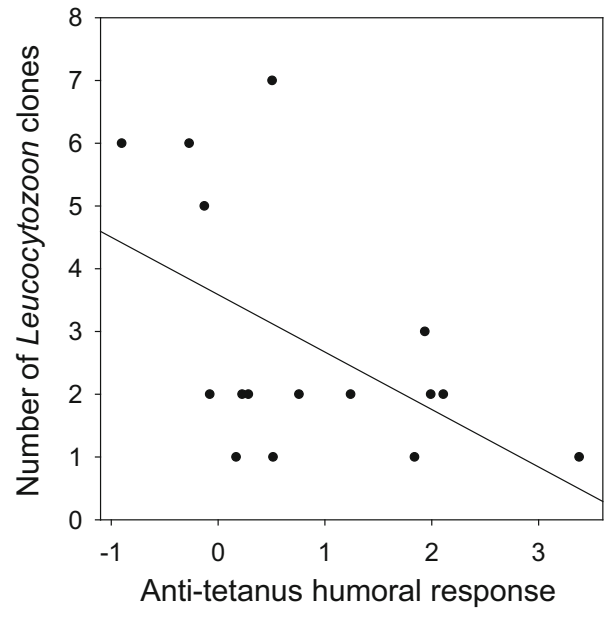

not dark but intermediate nestlings had the lowest PHA-response among all morphs. The PHA-induced swelling is the result of a complex cascade of immune signalling and many types of interacting cell types. This may convolute the effect of any potentially contributing melanisation-related genes. Additionally, the PHA response as a measure of skin inflammation did not mirror the pattern of ectoparasite distribution among nestling morphs (Chakarov et al. 2008). Neither did the PHA-response directly correlate with the actual ectoparasite infestation of the nestlings. This is not surprising since among different immune measures, ectoparasite infestation correlates most often with antibody and rarely with skin inflammation responses, without a clear direction of the latter correlations (Owen et al. 2010). Our results, however, indicate that intermediate buzzards might have the strongest anti-inflammatory reactions which might be selectively beneficial and contribute to their higher fitness later in life. 
Our prediction (2) was that the adaptive humoral response would be highest in dark nestlings. In contrast, we found that the humoral response in nestlings is explained by morph of their mothers and nestlings of intermediate mothers had the highest antibody production. Maternal effects on defences are mostly studied with respect to prenatal transfer of antibodies via yolk, and even postnatally via milk in mammals or other secretions in birds (Hasselquist and Nilsson 2009; Jacquin et al. 2012). It is generally assumed that wild birds are naïve towards vaccine antigens (e.g. Hanssen et al. 2005; Råberg et al. 2000), but maternal antibodies may unspecifically block antigens. This could inhibit the stimulation of specific antibody production in the first weeks of a nestling's life and create an opportunity for early incoming parasites such as Leucocytozoon (Staszewski and Siitari 2010). Thus, potentially, dark mothers could transfer the highest amount of antibodies to their nestlings. Unfortunately, experiments with vaccination of breeding females to reveal such an effect in our system have so far been impossible. Interestingly, most of the other studies comparing specific antibody production among colour polymorphic vertebrates also found a dependence on the maternal rather than on the own morph (Gasparini et al. 2009a; Jacquin et al. 2013; Roulin et al. 2000; Svensson et al. 2001). In side-blotched lizards Uta stansburiana, offspring of yellow-throated females have stronger anti-tetanus response than offspring of orange-throated females (Svensson et al. 2001). In barn owls Tyto alba, the antibody response increases with heritable plumage spottiness of the genetic mother, which also decreases the number and fecundity of ectoparasites (Roulin et al. 2000, 2001). In feral pigeons Columba livia, plumage darkness explains the transfer of specific antibodies against a natural antigen to the eggs (Jacquin et al. 2013). However, in Eleonora's falcons Falco eleonorae, a system most similar to the common buzzard polymorphism, not maternal but the own morph of nestlings explained their humoral response (Gangoso et al. 2015). The last example indicates that the developmental timing of the nestling immune system may differ between species and alter the effect of maternal morphs at the relative time of sampling.

Also in contrast to our prediction (2), a stronger humoral response was not associated with dark, but with intermediate morphs of the mothers. Intermediate buzzards have higher fitness and potentially higher parental quality. Therefore, they may provide more food resources, allowing higher investment into immune responses by their offspring (Møller and Petrie 2002). Indeed, heavier buzzard nestlings of given age were able to produce a stronger humoral response than lighter ones. Additionally, heterozygous intermediate females might transfer fewer non-specific maternal antibodies and thereby boost the production of specific antibodies in their offspring (Janeway et al. 2005; but see Boerner et al. 2013).

Overall, the production of a specific antibody response and inflammatory reactions are both expected to be costly and different morphs may have different investment strategies towards these defence axes. Our results, however, suggest that light and dark individuals are rather similar in both respects. Only intermediate morphs differed significantly in both antibody production and response to PHA, which cannot be explained by a simple functional or genetic (pleiotropic or linkage) correlation with melanisation. Thus, it might be plausible that a heterozygote advantage arises in intermediates through the interaction of immune-related genes belonging to light and dark morphs. This study, however, is correlative and the effects of plumage morph need to be verified and disentangled from environmental and parental effects through cross-fostering experiments and more prolonged measurements of the immune response development of nestlings (Gangoso et al. 2015). 
Antibody titres of nestlings explained their blood parasite infection status and genetic diversity, but not the intensity of infection. This finding supports the assumption that strong immune responses may effectively fight off parasites, limit the number of successful parasite strains, and is congruent with our prediction (3) (Hasselquist 2007). A common explanation is that parasite genetic diversity decreases with age because of stronger selection by the maturing host immunity (Bendixen et al. 2001). Indeed, we found a negative correlation between the genetic diversity of blood parasites and the strength of the humoral response, but also a weak increase of parasite diversity with host age, indicating that older nestlings may increasingly act as a "mixing bowl" for different parasite strains (Van den Broeck et al. 2014). Since more diverse infections may limit the potential of the immune system to react to novel antigens, the direction of a possible dependence remains to be established experimentally. Nevertheless, to our knowledge, this is the first report showing a correlation between the antibody response of a wild vertebrate and the genetic diversity of its blood parasites.

Genetically inherited colour polymorphisms are being increasingly associated with differences in parasitism and immunity (e.g. Calsbeek et al. 2008; Gangoso et al. 2011, 2015; Kerimov et al. 2012; Pryke et al. 2007). The exact mechanisms in melaninpolymorphic vertebrates are still unclear and possibly heterogeneous. Here we have shown that two anti-parasitic responses are morph-dependent and show exceptional levels for heterozygous intermediate morphs. A simple pleiotropy such as the POMC cascade may not be sufficient to explain such non-linear trends of immunity with melanisation (e.g. Calsbeek et al. 2008; Ducrest et al. 2008; Poelstra et al. 2014; Svensson et al. 2001). Such patterns may result from interactions between the genetic architecture and physiology behind colour polymorphisms, which need to be explored further.

Acknowledgements We would like to thank Thomas Grünkorn who climbed nests more than once specifically for this study. We thank Sandra Sköld Chiriac and Dennis Hasselquist for methodological advice. Alexandre Roulin and three anonymous referees provided much appreciated advice on improving this manuscript. We are grateful to Katrin Lehmann, Elke Hippauf und Nikolaus von Engelhardt for help in the lab analyses and to Rudy Jonker and Astrid Potiek for help in the field. N.C. is supported by the Volkswagen Foundation within its Evolutionary Biology initiative, grant I/84 196. O.K. holds a Heisenberg Professorship of the German Science Foundation (DFG, KR 2089/2-1).

Open Access This article is distributed under the terms of the Creative Commons Attribution 4.0 International License (http://creativecommons.org/licenses/by/4.0/), which permits unrestricted use, distribution, and reproduction in any medium, provided you give appropriate credit to the original author(s) and the source, provide a link to the Creative Commons license, and indicate if changes were made.

\section{References}

Bendixen M, Msangeni H, Pedersen BV, Shayo D, Bedker R (2001) Diversity of Plasmodium falciparum populations and complexity of infections in relation to transmission intensity and host age: a study from the Usambara Mountains, Tanzania. Trans R Soc Trop Med Hyg 95:143-148

Bensch S, Hellgren O, Pérez-Tris J (2009) MalAvi: a public database of malaria parasites and related haemosporidians in avian hosts based on mitochondrial cytochrome b lineages. Mol Ecol Resour 9:1353-1358

Boerner M, Krüger O (2009) Aggression and fitness differences between plumage morphs in the common buzzard (Buteo buteo). Behav Ecol 20:180-185

Boerner M, Hoffman JI, Amos W, Chakarov N, Krüger O (2013) Testing heterozygosity-fitness-correlations and inbreeding in colour morphs of different fitness in the common buzzard. J Evol Biol 26:2233-2243. doi:10.1111/jeb.12221 
Briggs CW, Collopy MW (2012) Extra-pair paternity in Swainson's Hawks. J Field Ornithol 83:41-46. doi:10.1111/j.1557-9263.2011.00354.x

Burnham KP, Anderson DR (2002) Model selection and inference: a practical information-theoretic approach, 2nd edn. Springer, Berlin

Caillaud D, Prugnolle F, Durand P, Théron A, de Meeûs T (2006) Host sex and parasite genetic diversity. Microbes Infect 8:2477-2483. doi:10.1016/j.micinf.2006.06.003

Calsbeek R, Bonneaud C, Smith TB (2008) Differential fitness effects of immunocompetence and neighbourhood density in alternative female lizard morphs. J Anim Ecol 77:103-109. doi:10.1111/j.13652656.2007.01320.x

Chakarov N, Boerner M, Krüger O (2008) Fitness in common buzzards at the cross-point of opposite melanin-parasite interactions. Funct Ecol 22:1062-1069

Chakarov N, Jonker RM, Boerner M, Hoffman JI, Krüger O (2013) Variation at phenological candidate genes correlates with timing of dispersal and plumage morph in a sedentary bird of prey. Mol Ecol 22:5430-5440. doi:10.1111/mec.12493

Chakarov N, Linke B, Boerner M, Goesmann A, Kruger O, Hoffman JI (2015a) Apparent vector-mediated parent-to-offspring transmission in an avian malaria-like parasite. Mol Ecol 24:1355-1363. doi:10. $1111 / \mathrm{mec} .13115$

Chakarov $\mathrm{N}$ et al (2015b) Territory quality and plumage morph predict offspring sex ratio variation in a raptor. PLoS ONE. doi:10.1371/journal.pone.0138295

Ducrest AL, Keller L, Roulin A (2008) Pleiotropy in the melanocortin system, coloration and behavioural syndromes. Trends Ecol Evol 23:502-510

Fowlie MK, Krüger O (2003) The evolution of plumage polymorphism in birds of prey and owls: the apostatic selection hypothesis revisited. J Evol Biol 16:577-583

Fridolfsson AK, Ellegren H (1999) A simple and universal method for molecular sexing of non-ratite birds. J Avian Biol 30:116-121. doi:10.2307/3677252

Gangoso L et al (2011) MC1R-dependent, melanin-based colour polymorphism is associated with cellmediated response in the Eleonora's falcon. J Evol Biol 24:2055-2063. doi:10.1111/j.1420-9101.2011. 02336.x

Gangoso L, Roulin A, Ducrest A-L, Grande JM, Figuerola J (2015) Morph-specific genetic and environmental variation in innate and acquired immune response in a color polymorphic raptor. Oecologia 178:1113-1123

Gasparini J et al (2009a) Strength and cost of an induced immune response are associated with a heritable melanin-based colour trait in female tawny owls. J Anim Ecol 78:608-616. doi:10.1111/j.13652656.2008.01521.x

Gasparini J, Piault R, Bize P, Roulin A (2009b) Pre-hatching maternal effects inhibit nestling humoral immune response in the tawny owl Strix aluco. J Avian Biol 40:271-278. doi:10.1111/j.1600-048X. 2008.04590.x

Gasparini J, Piault R, Bize P, Roulin A (2009c) Synergistic and antagonistic interaction between different branches of the immune system is related to melanin-based coloration in nestling tawny owls. J Evol Biol 22:2348-2353. doi:10.1111/j.1420-9101.2009.01831.x

Glutz von Blotzheim U, Bauer K, Bezzel E (1971) Handbuch der Vögel Mitteleuropas. Aula-Verlag, Wiesbaden

Hanssen SA, Hasselquist D, Folstad I, Erikstad KE (2005) Cost of reproduction in a long-lived bird: incubation effort reduces immune function and future reproduction. Proc R Soc B Biol Sci 272:1039-1046. doi:10.1098/rspb.2005.3057

Hasselquist D (2007) Comparative immunoecology in birds: hypotheses and tests. J Ornithol 148:S571S582. doi:10.1007/s10336-007-0201-X

Hasselquist D, Nilsson JA (2009) Maternal transfer of antibodies in vertebrates: trans-generational effects on offspring immunity. Philos Trans R Soc B Biol Sci 364:51-60. doi:10.1098/rstb.2008.0137

Hoi-Leitner M, Romero-Pujante M, Hoi H, Pavlova A (2001) Food availability and immune capacity in serin (Serinus serinus) nestlings. Behav Ecol Sociobiol 49:333-339

Jacquin L, Lenouvel P, Haussy C, Ducatez S, Gasparini J (2011) Melanin-based coloration is related to parasite intensity and cellular immune response in an urban free living bird: the feral pigeon Columba livia. J Avian Biol 42:11-15. doi:10.1111/j.1600-048X.2010.05120.x

Jacquin L, Blottière L, Haussy C, Perret S, Gasparini J (2012) Prenatal and postnatal parental effects on immunity and growth in 'lactating' pigeons. Funct Ecol 26:866-875. doi:10.1111/j.1365-2435.2012. 01988.x

Jacquin L, Haussy C, Bertin C, Laroucau K, Gasparini J (2013) Darker female pigeons transmit more specific antibodies to their eggs than do paler ones. Biol J Linn Soc 108:647-657. doi:10.1111/bij. 12001 
Janeway CAJ, Travers P, Walport M, Schlomchik MJ (2005) Immunobiology. Garland Science, New York Kerimov AB, Rogovin KA, Ivankina EV, Bushuev AV, Sokolova OV, Ilyina TA (2012) Specific immunity and polymorphism of breeding plumage in pied flycatcher (Ficedula hypoleuca) males (Aves: Passeriformes). Zhurnal Obshchei Biol 73:349-359

Krasnov BR, Mouillot D, Khokhlova IS, Shenbrot GI, Poulin R (2005) Covariance in species diversity and facilitation among non-interactive parasite taxa: all against the host. Parasitology 131:557-568

Krüger O (2002) Dissecting common buzzard lifespan and lifetime reproductive success: the relative importance of food, competition, weather, habitat and individual attributes. Oecologia 133:474-482

Krüger O, Lindström J, Amos W (2001) Maladaptive mate choice maintained by heterozygote advantage. Evolution 55:1207-1214

Lei BN, Amar A, Koeslag A, Gous TA, Tate GJ (2013) Differential haemoparasite intensity between black sparrowhawk (Accipiter melanoleucus) morphs suggests an adaptive function for polymorphism. PLoS ONE. doi:10.1371/journal.pone.0081607

Lochmiller RL, Deerenberg C (2000) Trade-offs in evolutionary immunology: just what is the cost of immunity? Oikos 88:87-98

Martin LB, Han P, Lewittes J, Kuhlman JR, Klasing KC, Wikelski M (2006) Phytohemagglutinin-induced skin swelling in birds: histological support for a classic immunoecological technique. Funct Ecol 20:290-299

McDade TW, Georgiev AV, Kuzawa CW (2016) Trade-offs between acquired and innate immune defenses in humans. Evol Med Public Health 2016:1-16. doi:10.1093/emph/eov033

Møller AP, Petrie M (2002) Condition dependence, multiple sexual signals, and immunocompetence in peacocks. Behav Ecol 13:248-253

Moret Y (2003) Explaining variable costs of the immune response: selection for specific versus non-specific immunity and facultative life history change. Oikos 102:213-216

Norris K, Evans MR (2000) Ecological immunology: life history trade-offs and immune defense in birds. Behav Ecol 11:19-26

Ntoumi F, Contamin H, Rogier C, Bonnefoy S, Trape JF, Mercereaupuijalon O (1995) Age-dependent carriage of multiple Plasmodium falciparum merozoite surface antigen-2 alleles in asymprtomatic malaria infections. Am J Trop Med Hyg 52:81-88

Owen JP, Nelson AC, Clayton DH (2010) Ecological immunology of bird-ectoparasite systems. Trends Parasitol 26:530-539. doi:10.1016/j.pt.2010.06.005

Poelstra JW et al (2014) The genomic landscape underlying phenotypic integrity in the face of gene flow in crows. Science 344:1410-1414. doi:10.1126/science.1253226

Pryke SR, Astheimer LB, Buttemer WA, Griffith SC (2007) Frequency-dependent physiological trade-offs between competing colour morphs. Biol Lett 3:494-497. doi:10.1098/rsbl.2007.0213

Råberg L, Nilsson JA, Ilmonen P, Stjernman M, Hasselquist D (2000) The cost of an immune response: vaccination reduces parental effort. Ecol Lett 3:382-386

Riley EM, Wahl S, Perkins DJ, Schofield L (2006) Regulating immunity to malaria. Parasite Immunol 28:35-49

Roulin A (2004) The evolution, maintenance and adaptive function of genetic colour polymorphism in birds. Biol Rev 79:815-848

Roulin A, Jungi TW, Pfister H, Dijkstra C (2000) Female barn owls (Tyto alba) advertise good genes. Proc R Soc Lond Ser B Biol Sci 267:937-941

Roulin A, Riols C, Dijkstra C, Ducrest AL (2001) Female plumage spottiness signals parasite resistance in the barn owl (Tyto alba). Behav Ecol 12:103-110

Rudnick JA, Katzner TE, Bragin EA, Rhodes OE, Dewoody JA (2005) Using naturally shed feathers for individual identification, genetic parentage analyses, and population monitoring in an endangered Eastern imperial eagle (Aquila heliaca) population from Kazakhstan. Mol Ecol 14:2959-2967. doi:10. 1111/j.1365-294X.2005.02641.x

Sandell MI, Tobler M, Hasselquist D (2009) Yolk androgens and the development of avian immunity: an experiment in jackdaws (Corvus monedula). J Exp Biol 212:815-822. doi:10.1242/jeb.022111

Schmid-Hempel P, Ebert D (2003) On the evolutionary ecology of specific immune defence. Trends Ecol Evol 18:27-32

Smits JE, Bortolotti GR, Tella JL (1999) Simplifying the phytohaemagglutinin skin-testing technique in studies of avian immunocompetence. Funct Ecol 13:567-572

Staszewski V, Siitari H (2010) Antibody injection in the egg yolk: maternal antibodies affect humoral immune response of the offspring. Funct Ecol 24:1333-1341. doi:10.1111/j.1365-2435.2010.01745.x

Sternalski A, Mougeot F, Bretagnolle V (2012) Phenotypic variation in nestlings of a bird of prey under contrasting breeding and diet conditions. Biol J Linn Soc 107:799-812. doi:10.1111/j.1095-8312.2012. 01981.x 
Svensson EI, Råberg L (2010) Resistance and tolerance in animal enemy-victim coevolution. Trends Ecol Evol 25:267-274. doi:10.1016/j.tree.2009.12.005

Svensson E, Raberg L, Koch C, Hasselquist D (1998) Energetic stress, immunosuppression and the costs of an antibody response. Funct Ecol 12:912-919. doi:10.1046/j.1365-2435.1998.00271.x

Svensson E, Sinervo B, Comendant T (2001) Density-dependent competition and selection on immune function in genetic lizard morphs. Proc Natl Acad Sci USA 98:12561-12565. doi:10.1073/pnas. 211071298

Van den Broeck F et al (2014) Inbreeding within human Schistosoma mansoni: do host-specific factors shape the genetic composition of parasite populations? Heredity 113:32-41. doi:10.1038/hdy.2014.13

Woolaver LG, Nichols RK, Morton ES, Stutchbury BJM (2013) Social and genetic mating system of Ridgway's hawk (Buteo ridgwayi), an endemic raptor on Hispaniola. J Trop Ecol 29:531-540. doi:10. $1017 / \mathrm{s} 0266467413000655$ 